\title{
Cost-Effectiveness of Emergent MRI during Stroke Alert to Diagnose Stroke Mimics: Single-Center Experience
}

\author{
Nidhi Kapoor ${ }^{1}$ Rohan Sharma ${ }^{1}$ Courtney Iser $^{2} \quad$ Kaustubh Chaudhari $^{3} \quad$ Krishna Nalleballe $^{1}$ \\ Aliza Brown ${ }^{1}$ Poornachand Veerapaneni ${ }^{1}$ Sen Sheng ${ }^{1}$ Hisham Elkhider ${ }^{1}$ \\ Karthika Veerapaneni ${ }^{1}$ Sanjeeva Onteddu ${ }^{1}$ Evgeny Sidorov ${ }^{2}$
}

${ }^{1}$ Department of Neurology, University of Arkansas for Medical

Sciences, Little Rock, Arkansas, United States

${ }^{2}$ Department of Neurology, University of Oklahoma Medical Science Center, Oklahoma City, Oklahoma, United States

${ }^{3}$ Department of Veterans Affairs Medical Center, Oklahoma City, Oklahoma, United States

\begin{abstract}
Address for correspondence Nidhi Kapoor, MD, Department of Neurology, University of Arkansas for Medical Sciences, 4301 W. Markham Street, Slot 500, Little Rock, AR-72205, United States (e-mail: nkapoor@uams.edu).
\end{abstract}

Abstract



Keywords
- acute ischemic stroke
management
- duration of
hospital stay
- emergent MRI
- health care cost
- ischemic stroke
- MRI
- stroke mimics

Objective The aim of this article was to assess the role of emergent magnetic resonance imaging (MRI) for stroke mimics during a stoke alert (within 45 minutes) in reducing direct cost of management and duration of inpatient stay.

Methods We did a retrospective chart review of all the patients who received emergent MRI brain during a stroke alert to help make decision about intravenous tissue-type plasminogen activator (IV tPA) administration from January 2013 to December 2015. Using the patient financial resource data and with the help of billing department, we calculated the approximate money saved in taking care of the patients who may have received IV tPA if emergent MRI brain was not available to diagnose stroke mimics as they presented with acute neurologic deficit within IV tPA time window.

Results Ninety seven out of 1,104 stroke alert patients received emergent MRI. Of these only 17 (17.5\%) were diagnosed with acute ischemic stroke (AIS), and 80 (82.5\%) as stroke mimics. By doing emergent MRI for suspected stroke mimics, our study showed an approximate total saving of $\$ 1,005,720$ to $\$ 1,384,560$, that is, $\$ 12,571$ to $\$ 17,307$ per patient in medical expenditure.

Discussion We suggest modification of stroke pathway from current algorithm " $C T+C T A \geq I V$-tPA/neurointervention $\geq M R I$ " to "MRI+MRA $\geq I V$-tPA/neurointervention" for possible stroke mimics, which can reduce the cost, radiation exposure, and duration of hospital stay for stroke mimics.

Conclusion Emergent MRI is a cost-effective tool to evaluate IV-tPA eligibility for suspected stroke mimics during a stroke alert.

\section{Introduction}

Stroke is the third leading cause of mortality globally ${ }^{1}$ and second leading cause of dementia, fourth leading cause of mortality and one of the leading causes of disability in the United States. ${ }^{2}$ Stroke risk increases considerably with

DOI https://doi.org/ 10.1055/s-0040-1721196 ISSN 0976-3147. advancing age, and it is estimated that in adults with age 35 to 44 , stroke incidence is 30 to 120 of 100,000 per year; and for those with age 65 to 74 , it increases to 670 to 970 of 100,000 per year. ${ }^{2}$. With increasingly aging population of United States and other countries, ${ }^{3,4}$ the burden of this disease 
is expected to rise. It was estimated in a study that in 2007 alone, cost of stroke was approximately $\$ 62.7$ billion. ${ }^{5} \mathrm{~A}$ recent meta-analysis by Demaerschalk et al assessed the short-term (in hospital) financial burden from stroke to range between $\$ 6,000$ and $\$ 23,000$ per admission, depending upon the disease course and complications during hospitalization. ${ }^{6}$ This cost was estimated after adjustment to 2008 Dollar.

"Stroke alert" or "Code stroke" is activated when a patient presents with acute neurologic deficit within a particular time window that makes them eligible for IV thrombolysis ${ }^{7-10}$ There are several clinical conditions such as seizures and migraines that can closely mimic a stroke in their presentation and often end up receiving the initial care for acute ischemic stroke (AIS) including intravenous tissue-type plasminogen activator (IV tPA) thus adding to stroke-related financial burden. Although the risk of intracranial hemorrhage (ICH) from IV tPA in these patients is low, ${ }^{11-13}$ avoiding IV thrombolysis is the best practice both from clinical and financial standpoint. We conducted this study to evaluate the role of emergent brain magnetic resonance imaging (MRI) in reducing direct cost of AIS management by avoiding unnecessary IV thrombolysis.

\section{Methods}

Primary objective of the study was to calculate amount of dollars saved by performing emergent brain MRI during stroke alert for suspected stroke mimics instead of directly administering IV tPA.

Stroke protocol at our institute: Stroke alert is activated when a patient presents with acute neurologic deficit within 7 hours. On call neurology resident obtains basic history, National Institutes of Health Stroke Scale (NIHSS), computed tomography (CT) head without contrast and computed tomography angiography (CTA) of head and neck, discusses with the neurology attending regarding IV tPA administration. Our institution can perform emergent brain MRI within 45 minutes, on request of stroke-neurology attending for suspected stroke mimics to aid in decision making for IV tPA administration. The first sequence obtained is diffusion-weighted imaging (DWI) and apparent diffusion coefficient (ADC). If the DWI is positive for AIS and correlates with ADC, the MRI is discontinued and the patient is given IV tPA.

Data collection and analysis: We identified patients who had emergent brain MRI during stroke alert from 2013 to 2015 using Comprehensive Stroke Center database and reviewed their charts. We collected clinical details (NIHSS, results of MRI, IV thrombolysis) and financial information (cost of IV tPA from our billing department and the approximate cost involved in taking care of an uncomplicated, uninsured stroke patient for 1 day from the patient financial resource data at our institute ${ }^{14}$ ). We carefully looked at the inclusion and exclusion criteria of administering IV tPA and calculated the number of stroke mimic patients who may have received IV TPA and thus would have been cared for as a stroke patient if emergent brain MRI was not available. We used these numbers to calculate the approximate amount of dollars saved by performing the emergent brain MRI during a stroke alert for these patients.

\section{Results}

Out of the 1,104 stroke alerts that were activated from January 2013 to December 2015, and 97 patients received emergent brain MRI during the stroke alert to aid in decision about IV tPA administration. The number of emergent brain MRIs performed increased from 11 in 2013 to 36 in 2014 and 50 in 2015. Out of the 97 patients that received emergent brain MRI, 80 patients (82.5\%) were diagnosed with a stroke mimic and 17 patients (17.5\%) with AIS. Of these 17 patients, 5 ended up getting IV tPA, and the reasons of not giving IV tPA to patients with MRI confirmed AIS are further delineated in -Table 1. The average door-to-needle time in the group that received emergent MRI brain was 80.8 minutes. Discharge diagnoses of the 80 stroke mimic patients are delineated in - Table 2. If we had not done emergent MRI, 87 of these 97 patients might have received IV tPA as they

Table 1 Reason for not giving IV tPA in patients with MRI-confirmed AIS

\begin{tabular}{|l|l|}
\hline Reasons & $\begin{array}{l}\text { Number of } \\
\text { patients }\end{array}$ \\
\hline $\begin{array}{l}\text { Bleeding from ETT after MRI brain was } \\
\text { completed }\end{array}$ & 1 \\
\hline $\begin{array}{l}\text { Initially MRI-ve, later found to have basilar } \\
\text { thrombus on repeat MRI }\end{array}$ & 1 \\
\hline $\begin{array}{l}\text { History of intracerebral hemorrhage found } \\
\text { during MRI brain }\end{array}$ & 1 \\
\hline Became outside time window due to MRI brain & 1 \\
\hline $\begin{array}{l}\text { NIHSS improved to } 1 \text { by the time MRI brain was } \\
\text { completed }\end{array}$ & 1 \\
\hline $\begin{array}{l}\text { Found to be outside time window on further } \\
\text { history that was determined at the time MRI } \\
\text { was being performed }\end{array}$ & 7 \\
\hline
\end{tabular}

Abbreviations: AIS, acute ischemic stroke; ETT, endotracheal tube; IV tPA; intravenous tissue-type plasminogen activator; MRI, magnetic resonance imaging; NIHSS, National Institutes of Health Stroke Scale.

Table 2 Common discharge diagnosis of stroke mimics

\begin{tabular}{|l|l|}
\hline Discharge diagnosis & $\begin{array}{l}\text { Number of } \\
\text { patients }\end{array}$ \\
\hline Hemiplegic migraine & 30 \\
\hline Toxic metabolic encephalopathy & 20 \\
\hline Seizure & 17 \\
\hline Transient ischemic attack & 5 \\
\hline Reactivation of old stroke symptoms & 3 \\
\hline Conversion disorder & 1 \\
\hline Lumbar strain & 1 \\
\hline Syncope & 1 \\
\hline Ramsay Hunt syndrome & 1 \\
\hline Benign paroxysmal positional vertigo & 1 \\
\hline History of spinal epidural hematoma & 1 \\
\hline
\end{tabular}


presented with an acute neurologic deficit within the appropriate time window, exception being the 7 patients who were eventually found to be outside time window at the time of presentation, 1 who had bleeding from endotracheal tube, 1 found to have history of ICH, and 1 who had rapidly improving exam. We calculated the approximate cost involved had the 80 stroke mimics received IV tPA. Cost of $100 \mathrm{mg}$ vial of IV tPA at our institution as found from billing department is $\$ 7897$ (X 80= $\$ 631,760$ ). Cost of 1 day of inpatient services for uncomplicated, unventilated stroke patient as determined from patient financial resources data declared for uninsured patients at our institution was between $\$ 4674.5$ $(X 80=\$ 373,960)$ and $\$ 9,410(X 80=\$ 752,800)$. The cost of MRI brain was not included in this analysis as an MRI would eventually be obtained in all patients to confirm or rule out AIS. At our institute, the cost of emergent MRI does not significantly differ from that of nonemergent MRI. Adding the cost of IV TPA and inpatient stay, approximate savings by doing MRI brain emergently during the stroke alert for these patients were found to between $\$ 1,005,720$ (\$631,760 $+\$ 373,960)$ and $\$ 1,384,560(\$ 631,760+\$ 752,800)$, that is, between $\$ 12,572$ and $\$ 17,307$ per patient.

\section{Discussion}

Our study showed that cost of administering IV tPA to patient with possible stroke mimic exceeds the opportune cost of emergent MRI. High-volume stroke centers see high rates of stroke mimics. These rates can be as high as 6 to $14 \%$ in some centers. $^{15-18}$ Also, stoke mimics treated with IV tPA varies widely from 1.4 to $16.7 \%$ between different institutes. ${ }^{19-21}$ Risk of intracranial bleeding after IV tPA is relatively low in patients presenting as stroke mimics. ${ }^{11-13}$ Despite low risk of ICH, it remains a major concern for IV tPA administration. IV tPA increases the cost of care for stroke mimic patients considerably, which includes the cost of the drug and hospital stay following the drug administration. Our study showed a reduction of $\$ 12,571$ to $\$ 17,307$ per patient in medical expenditure for patients presenting with stroke mimics.

Various studies have shown the feasibility of using specific MRI sequences as part of acute stroke protocols in patients eligible for IV tPA. ${ }^{22}$ We propose that by modifying stroke pathway from algorithm I [CT+CTA $\geq I V$ tPA \pm neuro-intervention $\geq$ MRI] to algorithm II [Emergent MRI+MRA $\geq$ IV tPA \pm Neurointervention], we can further reduce the cost in stroke pathways by $\$ 1,476$ to $\$ 4,176$ per patient by eliminating the cost of CT that was not calculated in our study.

\section{Limitations of the Study}

Our study has certain limitations. It is a retrospective chart review with a small sample size. There is also physician bias for performing emergent brain MRI. There is always a concern that getting MRI even though emergent might delay the administration of IV tPA and along with a possibility that MRI could miss AIS especially in the early hyperacute phase-like the one patient that presented with negative MRI and later was discovered to have basilar thrombus. The costs that we included for calculation are on the lower side of the actual costs. This is because the cost of IV TPA and of the inpatient stay has increased since 2013 to 2015 so we approximated the cost. Also, the destination inpatient stay after IV tPA at our institute for a stroke patient is a stroke unit that is a dedicated unit on ward where level of care is a step down from intensive care unit (ICU) and a step above the regular neurology ward, thus allowing closer monitoring of these patients without incurring the increased cost of ICU admission on the patients. Most of the institutes in United States admit the patients to ICU after IV tPA administration that would further increase the cost involved on taking care of these patients.

\section{Conclusion}

We conclude that if stroke mimic is suspected on history and examination, then emergent brain MRI is a cost-efficient tool if done in a timely manner. By ruling out AIS and preventing unnecessary IV tPA administration, it helps to reduce the health care cost and the duration of inpatient hospital stay, especially at the institutions where MRI brain is readily available. Due to the above-mentioned limitations, further larger studies are needed.

\section{Funding}

None.

\section{Conflict of Interest}

None declared.

\section{References}

1 Murray CJ, Lopez AD. Mortality by cause for eight regions of the world: Global Burden of Disease Study. Lancet 1997;349(9061):1269-1276

2 Roger VL, Go AS, Lloyd-Jones DM, et al; American Heart Association Statistics Committee and Stroke Statistics Subcommittee. Heart disease and stroke statistics-2011 update: a report from the American Heart Association. Circulation 2011;123(4):e18-e209

3 World's older population grows dramatically. Online. National Institutes of Health (NIH); 2016. Available at: https:// www.nih.gov/news-events/news-releases/worlds-olderpopulation-grows-dramatically

4 Wan He DG, Paul Kowal. An Aging World: 2015. In: U.S. Department of Health and Human Services, National Institutes of Health and AGING. NIO, (eds.) International Population Reports United States Census Bureau website; 2016. (web site). Available at: https://www.census.gov/library/publications/2016/demo/P95-16-1.html

5 Rosamond W, Flegal K, Friday G, et al; American Heart Association Statistics Committee and Stroke Statistics Subcommittee. Heart disease and stroke statistics2007 update: a report from the American Heart Association Statistics Committee and Stroke Statistics Subcommittee. Circulation 2007;115(5):e69-e171

6 Demaerschalk BM, Hwang HM, Leung G. US cost burden of ischemic stroke: a systematic literature review. Am J Manag Care 2010;16(7):525-533 
7 Nalleballe K, Sharma R, Brown A, et al. Ideal telestroke time targets: Telestroke-based treatment times in the United States stroke belt. J Telemed Telecare 2020;26(3):174-179

8 Kim A, Lee JS, Kim JE, et al. Trends in yield of a code stroke program for enhancing thrombolysis. J Clin Neurosci 2015; 22(1):73-78

9 Hoegerl C, Goldstein FJ, Sartorius J. Implementation of a stroke alert protocol in the emergency department: a pilot study. J Am Osteopath Assoc 2011;111(1):21-27

10 Nalleballe K, Sharma R, Kovvuru S, et al. Why are acute ischemic stroke patients not receiving thrombolysis in a telestroke network? J Telemed Telecare 2020;26(6):317-321

11 Zinkstok SM, Engelter ST, Gensicke H, et al. Safety of thrombolysis in stroke mimics: results from a multicenter cohort study. Stroke 2013;44(4):1080-1084

12 Nguyen PL, Chang JJ. Stroke mimics and acute stroke evaluation: clinical differentiation and complications after intravenous tissue plasminogen activator. J Emerg Med 2015;49(2):244-252

13 Sivakumaran P, Gill D, Mahir G, Baheerathan A, Kar A. A retrospective cohort study on the use of intravenous thrombolysis in stroke mimics. J Stroke Cerebrovasc Dis 2016;25(5):1057-1061

14 Pricing Estimates and Information - Uninsured Patients. Patient Financial Resource. Online: OU Medical Center

15 Chernyshev OY, Martin-Schild S, Albright KC, et al. Safety of tPA in stroke mimics and neuroimaging-negative cerebral ischemia. Neurology 2010;74(17):1340-1345
16 Tsivgoulis G, Alexandrov AV, Chang J, et al. Safety and outcomes of intravenous thrombolysis in stroke mimics: a 6-year, single-care center study and a pooled analysis of reported series. Stroke 2011;42(6):1771-1774

17 Sarikaya H, Yilmaz M, Luft AR, Gantenbein AR. Different pattern of clinical deficits in stroke mimics treated with intravenous thrombolysis. Eur Neurol 2012;68(6):344-349

18 Förster A, Griebe M, Wolf ME, Szabo K, Hennerici MG, Kern R. How to identify stroke mimics in patients eligible for intravenous thrombolysis? J Neurol 2012;259(7):1347-1353

19 Hand PJ, Kwan J, Lindley RI, Dennis MS, Wardlaw JM. Distinguishing between stroke and mimic at the bedside: the brain attack study. Stroke 2006;37(3):769-775

20 Tobin WO, Hentz JG, Bobrow BJ, Demaerschalk BM. Identification of stroke mimics in the emergency department setting. J Brain Dis 2009;1:19-22

21 Brunser AM, Illanes S, Lavados PM, et al. Exclusion criteria for intravenous thrombolysis in stroke mimics: an observational study. J Stroke Cerebrovasc Dis 2013;22(7):1140-1145

22 Paolini S, Burdine J, Verenes M, et al. Rapid short MRI sequence useful in eliminating stroke mimics among acute stroke patients considered for intravenous thrombolysis. J Neurol Disord 2013;1:137 